\title{
Factores que limitan la participación de la inversión privada en el centro histórico de la ciudad de Veracruz
}

\section{Factors that limit the participation of private investment in the historical center of the City of Veracruz, Mexico}

\author{
DANiel VÁzQuez-Cotera \\ ELENA Moreno-García*
}

\begin{abstract}
For a decade the local government of Veracruz has undertaken important actions to foster the revitalization of its historical center; with these projects it intends to encourage the population to restore, buy or sell real estate in the area. Nonetheless, thus far the incentives to achieve this have turned out insufficient to attract private investment. Before this situation, in the present document, we analyze the factors that determine private participation in urban revitalization processes in order to evaluate the strategy of the local authority. The results show it is convenient to restate some actions to increase the chance of private participation, since nowadays both fundamental aspects of risk and returns associated to decisions are not considered.
\end{abstract}

Keywords: revitalization of the historical center; barriers to private participation; fiscal incentives.

\section{Resumen}

Desde hace una década el gobierno local de Veracruz ha realizado esfuerzos importantes para impulsar la revitalización de su centro histórico. Con estos proyectos se intenta generar incentivos para que a la población le resulte atractivo restaurar, comprar o vender bienes inmuebles de esa zona. Sin embargo, hasta ahora dichos incentivos han sido insuficientes para atraer la inversión privada. Ante esta situación, en el presente documento se analizan los factores que determinan la participación privada en procesos de revitalización urbana, para con ello evaluar la estrategia de la autoridad local. Los resultados muestran que es conveniente replantear algunas acciones para incrementar las posibilidades de participación privada, ya que actualmente no se contemplan aspectos fundamentales de riesgo y rendimiento asociados con las decisiones de inversión espacial.

Palabras clave: revitalización del centro histórico, barreras a la participación privada, incentivos fiscales.

*Universidad Cristóbal Colón, Veracruz, México. Correos-e: dcotera@aix.ver.ucc.mx, elenam@ aix.ver.ucc.mx. 


\section{Introducción ${ }^{1}$}

En el mes de septiembre de 2005 se publicó el decreto por el que se otorgan estímulos fiscales para el rescate de las zonas de monumentos históricos de los centros históricos de Mérida, Yucatán; Morelia, Michoacán, y la ciudad y puerto de Veracruz. Estos estímulos, a través de generar ahorros en el pago del impuesto sobre la renta (ISR), tenían el propósito de coadyuvar en la restauración y rehabilitación de los bienes inmuebles ubicados en los centros históricos de estas ciudades, produciendo incentivos para que a la población le resultara atractivo restaurar, comprar o vender bienes inmuebles de esa zona.

En el caso particular de Veracruz, estos incentivos generaron altas expectativas entre los funcionarios públicos locales, ${ }^{2}$ quienes llegaron a concebirlos como un complemento importante de los planes y proyectos de revitalización que el gobierno de la ciudad puso en práctica a partir de 1998. Sin embargo, hasta ahora, aun con dichos incentivos, las acciones realizadas son insuficientes para atraer la inversión privada, pues a tres años de vigencia del decreto, no hay un incremento significativo en la compra, venta, reparación y rehabilitación de los inmuebles de su centro histórico.

Si bien los procesos de renovación urbana implican un horizonte de tiempo de largo plazo, ante la escasa respuesta de la iniciativa privada se presentan algunas dudas sobre la conveniencia de la estrategia, y más aún en cuanto al aspecto fiscal, ya que después de la publicación de los incentivos también se produjeron una serie de modificaciones al marco legal, en particular con la incorporación del impuesto especial sobre tasa única (IETU), que incrementan la incertidumbre sobre su efectividad.

Las experiencias en países de Europa y América han mostrado que, dada la restricción presupuestal de los gobiernos locales y la gran cantidad de recursos que se requiere para alcanzar el objetivo de la renovación urbana, la participación de la iniciativa privada resulta fundamental, por tanto, entender muy bien las motivaciones de su inserción en este tipo de proyectos es indispensable y permite evaluar qué tan adecuadas son las estrategias del sector público.

Por lo anterior, y para atender las dudas suscitadas en la experiencia de la ciudad de Veracruz, en este artículo se presenta un análisis sobre los factores que afectan la participación de la inversión privada en los proce-

\footnotetext{
${ }^{1}$ Los autores agradecen a las autoridades del H. Ayuntamiento de Veracruz y de la Universidad Cristóbal Colón por las facilidades otorgadas para la elaboración de este proyecto.

${ }^{2}$ Ésta fue la opinión que externaron los principales funcionarios de la Dirección del Centro Histórico del Ayuntamiento de Veracruz, dependencia encargada de favorecer e impulsar la revitalización de esa zona específica (entrevista, julio de 2008).
} 
sos de revitalización urbana. En específico se busca responder a las siguientes interrogantes: ¿¿cuál es el papel de la inversión privada en los procesos de renovación urbana?, ¡cuáles son los principales determinantes de su participación?, ¿cuál es el papel de los incentivos fiscales, qué se puede esperar de ellos y qué tan atractivos resultan aquéllos que aplican para mejorar el centro histórico de la ciudad de Veracruz?

\section{Metodología}

Para responder estas preguntas se llevó a cabo una revisión exhaustiva de fuentes secundarias -artículos publicados, informes publicados e inéditos, así como estadísticas de diversas fuentes- que dan cuenta de diversas experiencias internacionales en las que se han aplicado estrategias de revitalización urbana, así como de argumentos teóricos que justifican o critican las distintas estrategias. Para identificar los determinantes principales de la inversión privada en Veracruz, se realizaron entrevistas a personajes clave y a quienes toman decisiones en el sector público (del Instituto Nacional de Antropología e Historia y de la Dirección del Centro Histórico de Veracruz) y privado (desarrolladores, empresas inmobiliarias, inversionistas, hoteleros y comerciantes con intereses reales dentro del centro histórico). En total se realizaron 24 entrevistas individualizadas y semiestructuradas, de las cuales cuatro se hicieron a funcionarios públicos y el resto a informantes calificados de diversos sectores de la iniciativa privada.

En las entrevistas se intentó abordar el tema de los incentivos fiscales, pero como hasta ahora dichos incentivos no se han aplicado, se determinó que las respuestas sólo estarían basadas en especulaciones y, por lo mismo, se descartó utilizarlas. En lugar de ello, para analizar el atractivo de los incentivos fiscales en Veracruz se consideró conveniente seguir la propuesta metodológica de Peters y Fischer (2002) y de Papke (1995), entre otros, que consiste en hacer microsimulaciones sobre decisiones de ubicación de empresas hipotéticas. Para ello se diseñó una simulación financiera que compara dos inversiones del mismo monto y tipo, aplicadas en la adaptación de bienes inmuebles adquiridos en diferentes zonas de la ciudad: en el centro histórico y la periferia. Este análisis contempla tres escenarios con diferentes niveles de ventas cada uno, y refleja distintos resultados de acuerdo con la ubicación urbana de los proyectos y de las condiciones fiscales que le corresponderían a cada zona.

\section{Intervención pública reciente en el centro histórico de Veracruz}

El centro histórico es un espacio urbano conformado por un conjunto de relaciones sociales que lo configuran como eje de la ciudad y de su histo- 
ria (Carrión, 2000). Así definido es posible distinguir tres aspectos esenciales relacionados con el concepto de centro histórico: 1) lo espacial, ya que el centro se concibe como un lugar o escenario que tiene el atributo de la centralidad histórica; 2) el tiempo, porque los centros históricos condensan el proceso histórico de una ciudad y muestran el incremento del valor producido en la zona; y 3) lo patrimonial, pues en ellos aparece con notable peso la noción de herencia o legado en forma de patrimonio.

El centro histórico de la ciudad de Veracruz conserva vestigios de más de tres siglos de historia que muestran igualmente la evolución socioeconómica y arquitectónica de la ciudad. En él se encuentran la sede del Poder Ejecutivo del gobierno local y los inmuebles religiosos y culturales más representativos de la ciudad. Además, alberga a las compañías más importantes relacionadas con las actividades del puerto de Veracruz, ya que colinda con el mismo. Hasta hace apenas 20 años era el principal foco comercial de la mancha urbana. En la actualidad comprende un polígono de aproximadamente 164.8 ha que representa 5\% del área urbana de la zona metropolitana: Veracruz-Boca del Río (mapa I). Para fines oficiales dicho espacio lo constituyen los perímetros A, B y C delimitados, los dos primeros, en el decreto del gobierno federal publicado el 24 de marzo de 2004, en el que se le declaró zona de monumentos con fines de preservación, y el último, con fines de contención, se definió en los dos últimos planes que se han realizado sobre el área. En el último plan de revitalización urbana se estimó que en 2006, en conjunto los tres perímetros sumaban 5,526 predios, de los cuales $98 \%$ eran de propiedad privada y el resto públicos. Además se calculó que en 2005 en dicha zona habitaban alrededor de 17,000 personas, equivalente a $2.8 \%$ de la población del área conurbada. Por otro lado, entre los diversos usos predominan el comercial y el habitacional; en relación con su superficie neta, el primero ocupa $42 \%$ y el segundo $37 \%$. Los usos comerciales y mixtos prevalecen en el perímetro a y los habitacionales en los dos restantes.

Si bien la variedad de usos en el centro histórico hace evidente que es un espacio urbano consolidado, desde hace más de dos décadas se observa una marcada tendencia de despoblamiento, construcciones en estado ruinoso y un franco proceso de abandono del patrimonio arquitectónico. Esto motivó que el gobierno del estado y del municipio de Veracruz emprendieran un conjunto de proyectos para intentar revertir estas tendencias, los cuales quedaron plasmados en dos planes de revitalización urbana; el primero publicado en 1998 y el segundo en $2006 .^{3}$

\footnotetext{
${ }^{3}$ Programa de Ordenamiento, Mejoramiento y Revitalización del Centro Histórico de Veracruz (2006) y Programa de Ordenamiento y Revitalización del Centro Histórico de la Ciudad de Veracruz (1998).
} 


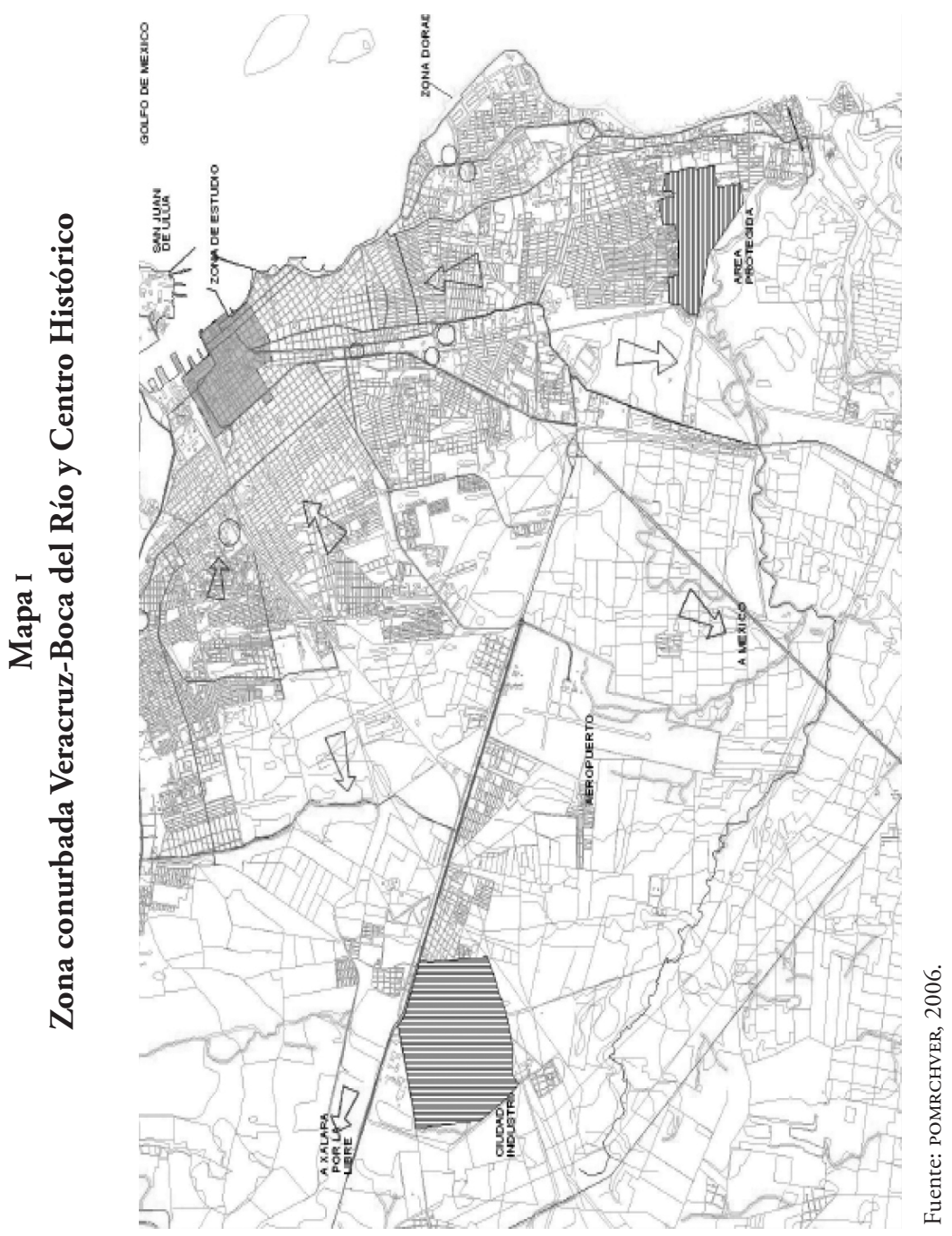


Estos planes tienen como objetivo conservar el centro histórico como un núcleo urbano polifuncional, en óptimas condiciones para las actividades turísticas, culturales y habitacionales. En ambos planes se propusieron diferentes acciones públicas que pretendían regular los usos de los inmuebles, mejorar las condiciones de acceso vehicular y de circulación peatonal, así como hacer más atractiva la zona; por ejemplo, entre las propuestas destacaba la construcción de un puente que se esperaba permitiría redireccionar el flujo vehicular y disminuir los niveles de congestión, además de ampliar los espacios para estacionamiento. En cuanto a las acciones para hacer más atractivo el recorrido de los peatones, se recomendó arreglar las fachadas, mejorar el mobiliario urbano (jardineras y bancas) y ampliar las banquetas en algunas de las principales calles y avenidas.

Se esperaba que estas obras lograran incentivar a la iniciativa privada para invertir en la zona y modificar así la tendencia del mercado inmobiliario. Sin embargo, como se mencionó, dicha participación ha sido marginal, por lo que se requiere identificar y evaluar los aspectos que pueden estar limitando los alcances de los proyectos. En las siguientes secciones se aborda este asunto de manera detallada.

\section{Participación pública y privada en los procesos de renovación urbana}

Es común que los centros históricos, a pesar de que están bien dotados de infraestructura, contengan una gran cantidad de edificios con diversos grados de abandono o subutilización y terrenos vacíos, lo cual es reflejo de una reducción de la cantidad de personas que lo habitan, y de una pérdida paulatina de su importancia relativa en materia comercial y de servicios ante el desarrollo de modernos complejos comerciales en zonas periféricas de la mancha urbana.

El despoblamiento es particularmente dañino ya que detona un círculo vicioso, toda vez que el abandono que sucede a la pérdida de población se traduce en un incremento del deterioro, y en la consiguiente disminución en el valor de las propiedades, ${ }^{4}$ lo que facilita a su vez la instalación de otras actividades, que por su naturaleza generan externalidades negativas que afectan el entorno y la calidad de vida residencial. Aunque cabe aclarar que en ocasiones es precisamente la especulación y la búsqueda de mayores rentas de corto plazo, lo que motiva que los

\footnotetext{
${ }^{4}$ En el caso particular de Veracruz, de acuerdo con un levantamiento de información de las principales inmobiliarias de la ciudad, este fenómeno se presenta principalmente en aquellos inmuebles destinados a vivienda, donde los precios de renta y venta llegan a ser hasta cuatro veces menores que los que se observan en otras áreas de la zona conurbada (Veracruz-Boca del Río).
} 
propietarios cambien el uso de los inmuebles, esto resta espacios habitacionales y afecta el potencial de la zona en el largo plazo.

En algunos centros históricos de América Latina el despoblamiento se ha dado con mayor intensidad, ya que el incremento del número de vehículos y la carencia de lugares para estacionarse contribuye a que muchos propietarios prefieran demoler sus inmuebles para hacerlos más rentables, convirtiéndolos en estacionamientos públicos que funcionan sobre todo por la mañana, pues por la tarde los centros se quedan casi vacíos generando en los transeúntes la sensación de inseguridad, independientemente que ésta se sostenga o no en hechos delictivos reales (Carrasco, 2007).

Estas circunstancias han orillado al sector público a intervenir para revertir el deterioro de dichas zonas, justificándose a partir de argumentos basados en aspectos culturales y/o económicos. Es común que se resalte la necesidad de conservar el patrimonio arquitectónico de la ciudad (Vázquez, 2007), sin embargo, también se ha encontrado (Ihlandfeldt, 1995) que las condiciones en estas áreas pueden afectar el potencial de desarrollo del resto de la ciudad, e incluso de la región, ya que, entre otras cosas, para los visitantes foráneos la zona puede ser una referencia general de la ciudad o región, por lo que su estado puede determinar la percepción sobre el atractivo de ambas y el interés por visitarlas. Otro aspecto, menos común, que se resalta es el hecho de que el deterioro económico de la zona central también puede afectar el potencial recaudatorio de las autoridades locales, lo cual genera incentivos para compensar la caída de los ingresos públicos mediante el traslado de la carga del presupuesto a otras zonas de la ciudad, generando así un incremento en las distorsiones de las decisiones económicas que reducen el potencial global de crecimiento de toda la ciudad.

En general se ha observado que las estrategias implementadas en diversos países han tenido en común el objetivo de promover el mejor uso en términos sociales y económicos de una zona urbana que padece deterioro social, económico y físico e infrautilización de sus activos, edificios, suelo, infraestructura o espacios públicos (Rojas, 2004; Espinosa, 2004; Leal 2004; entre otras experiencias documentadas). En algunas ciudades de países como España, Irlanda, Chile, Argentina, México y Colombia, la intervención gubernamental ha implicado la aplicación de una o varias de las siguientes acciones:

- Compra de edificios para conservarlos y aprovecharlos con proyectos de beneficio social o para revenderlos a la iniciativa privada, una vez que se ha demostrado su potencial económico. 
- Implementación de regulaciones para prevenir la destrucción de los inmuebles patrimoniales por uso inadecuado.

- Inversión en la educación de la comunidad en relación con el significado de la herencia patrimonial de su ciudad.

- Inversión en mejoramiento de la infraestructura e imagen urbana, con el objetivo de mejorar tanto las condiciones de atractivo como de acceso.

- Otorgamiento de subsidios, desde la asesoría técnica hasta las transferencias directas de capital.

- Diseño e implementación de incentivos fiscales (totales o parciales) para motivar a los propietarios y a los inversionistas en general a preservar los activos patrimoniales, compensándoles el costo en el que hubieran incurrido para lograrlo.

Así, además de preocuparse por la conservación arquitectónica, la intervención pública también procura reducir las barreras que limitan la participación de la inversión privada, la cual tiene un papel determinante utilizando sus recursos para coadyuvar en la creación de conceptos inmobiliarios que permiten elevar el valor de las propiedades, mejorar las expectativas y, en general, hacer sostenible todo el proceso de renovación. De hecho, dado que los recursos públicos son escasos y que en zonas con deterioro urbano el nivel de eficiencia del mercado inmobiliario es bajo, actualmente se considera prioritario que la estrategia se base en reducir las barreras de acceso a la iniciativa privada, ya que de lo contrario no participaría y se pondría en riesgo el financiamiento de todo el proyecto (Adair et al., 2002).

Si bien la revitalización es un proceso complejo, se han identificado cuando menos tres etapas de desarrollo (Adair et al., 2006) en las que la iniciativa privada puede participar. La etapa inicial contempla un proceso de regeneración de los espacios urbanos que normalmente requiere de inversión para mejorar las condiciones de infraestructura y, por tanto, de cuantiosos recursos financieros. Por lo mismo, en países desarrollados como Estados Unidos y Gran Bretańa, desde hace ya algunas décadas, se han diseñado instrumentos de deuda que permiten financiar esta etapa, mediante su colocación entre el público inversionista (McGreal et al., 2002). En experiencias de otros países como Argentina, en Puerto Madero (Rojas, op. cit.), y España, en Bilbao (Rodríguez y Martínez, 2001), los gobiernos locales o agencias especiales en favor del desarrollo de proyectos de renovación urbana, obtuvieron donaciones de activos importantes o lotes de grandes dimensiones que les permitieron adquirir los recursos para iniciar el proceso. 
La segunda fase consiste en restaurar y acondicionar los inmuebles para los distintos fines que hayan sido considerados como viables y convenientes. En esta fase es en la que comúnmente se han centrado los incentivos públicos (por ejemplo, exenciones fiscales y permisos transferibles de desarrollo) para atraer a desarrolladores de la iniciativa privada, teniendo en cuenta que como cualquier proyecto privado, las decisiones de invertir están en función del rendimiento y riesgo que puedan lograr. En algunos países se han constituido también empresas públicas, fideicomisos o trusts que se financian con fondos privados, reduciendo de esta manera el riesgo al favorecer las condiciones de salida de los inversionistas. Finalmente, la tercera etapa consiste en vender los inmuebles al público inversionista e interesados en general, en un mercado transformado, más dinámico y eficiente.

Como corolario, se puede argumentar que si bien los centros históricos siguen siendo uno de los lugares más emblemáticos de las zonas urbanas y cuentan con un potencial de desarrollo importante, ello no representa un estímulo suficiente para que la iniciativa privada dé el primer paso en el proceso de renovación, sin embargo, su participación es determinante y, por lo mismo, la estrategia del gobierno debe procurarla, creando para ello proyectos innovadores que incidan en la perspectiva del riesgo y el rendimiento de la inversión en la zona, en comparación con lo que se puede obtener en otras ubicaciones de la ciudad en todas las etapas del proyecto de desarrollo.

\section{Determinantes de la participación de la iniciativa privada}

Si se acepta la premisa de que la estrategia de revitalización requiere de la inversión privada para ser sostenible, entonces es fundamental entender cómo funciona el mercado inmobiliario, ya que ello permitirá comprender en qué circunstancias a la iniciativa privada le podría resultar atractivo participar en el proyecto del centro histórico. Para esto, el objetivo debe centrarse en conocer las motivaciones de los inversionistas y empresas desarrolladoras que pudieran poner en marcha los proyectos, así como la de los demandantes o usuarios finales de los inmuebles, tanto para el caso de viviendas como para negocios.

En este sentido, diversos estudios han mostrado que el factor principal que motiva la participación de la inversión privada en un proyecto de regeneración, es la percepción de alcanzar un nivel de rendimiento superior al que se podría obtener en promedio en proyectos inmobiliarios alternativos (Adair et al., 2006; McGreal et al., 2000, entre otros). De hecho, se ha encontrado que el crecimiento potencial de las rentas, motivado por la dinámica de la demanda de las propiedades, y el crecimien- 
to esperado del valor de los activos, motivado por el comportamiento de la demanda de los inversionistas, son los factores primordiales utilizados en la evaluación de este tipo de proyectos. Por lo mismo, es común que entre las principales limitantes a la participación privada se encuentren las expectativas pesimistas sobre el rendimiento de la inversión, asociadas a percepciones sobre bajos niveles de apreciación y de rentas de las propiedades, que en buena parte son consecuencia de señales confusas de un mercado en el que existen dificultades para obtener información confiable y a un costo asequible (Berry et al., 2003).

Otras barreras comunes se relacionan con el grado de deterioro físico y/o social de la zona, y los retrasos de las autoridades locales para otorgar permisos de construcción o remodelación. Por otro lado, dado que el proceso de renovación implica la participación del sector público por un lapso que supera al de una administración (en los diferentes niveles de gobierno), otro desincentivo importante es la percepción sobre la falta del compromiso suficiente de las autoridades con el desarrollo de todo el proyecto. El mismo hecho de que se trate de un proyecto de largo plazo impone el riesgo de que éste atraviese por una etapa adversa de un ciclo económico, y que la demanda por las propiedades se reduzca significativamente.

Cabe resaltar que el efecto de los desincentivos es diferente en cada proyecto de revitalización, y por lo mismo se consideró conveniente realizar un conjunto de entrevistas a un grupo de inversionistas potenciales (todos de Veracruz), para que desde su perspectiva comentaran cuáles consideran son las barreras más importantes a la inversión inmobiliaria en el centro histórico de la ciudad de Veracruz. Entre las personas entrevistadas hay propietarios de empresas desarrolladoras, inmobiliarias, hoteles y comercios en general que tienen en común ser inversionistas potenciales con intereses actuales en la zona.

Además de que todos coinciden en que es en otras áreas de la zona metropolitana donde se perciben mejores condiciones de inversión, la mayoría ( $80 \%$ de los entrevistados) también señala a las instituciones y los trámites que regulan el desarrollo dentro del centro histórico (el Instituto Nacional de Antropología e Historia y a la Dirección del Centro Histórico del Ayuntamiento) como las principales barreras, pues los trámites que entre otras cosas tratan de garantizar la conservación de los bienes inmuebles, se vuelven un proceso largo y costoso, no tanto por el pago de los permisos y las licencias que se solicitan, sino por el costo de oportunidad de la inversión durante los meses que se lleva dar cumplimiento a los requisitos de dichas dependencias, y con la incertidumbre de que no se otorgue.

Siete entrevistados argumentaron que aun en proyectos impulsados por la misma autoridad municipal que representaban inversiones importantes, 
se han presentado dificultades de trámites entre dependencias públicas, lo cual daña significativamente la percepción de los empresarios sobre la capacidad de gestión de las autoridades locales; fue común que aludieran a dichos proyectos para señalar la problemática de hacer negocios en el centro histórico y la incapacidad de las autoridades para favorecerlos.

Si bien sólo un entrevistado lo mencionó, otro determinante importante es la condición legal de buena cantidad de propiedades de la zona. Muchas de las que estaban arrendadas se encuentran en litigios que llevan ya muchos años sin resolución. Este detalle imprime un riesgo singular, ya que propiedades en estas condiciones no son sujetas a comercializarse bajo ninguna circunstancia.

Por otro lado, los cuatro empresarios más importantes del sector inmobiliario en Veracruz, comentaron que, sobre todo en el caso de viviendas y oficinas, la demanda en el centro es escasa, lo que genera que la rapidez de comercialización sea baja en comparación con otras áreas de la zona conurbada; por ejemplo, inmuebles en renta para oficinas pueden tardar entre cinco y nueve meses para comercializarse, mientras que en la zona sur de la ciudad en promedio se tardan entre dos y tres meses. Para la vivienda las diferencias son mayores; en el centro histórico se tardan más de un año en rentarlas, mientras que en otras áreas en promedio tardan sólo entre tres y cinco meses.

Es importante apuntar que $90 \%$ de las entrevistados coincidieron también en que la principal desventaja del centro histórico se relaciona con las condiciones de acceso, que tienen que ver con el congestionamiento vehicular y los espacios de estacionamiento, lo cual puede, en parte, explicar el poco atractivo de los inmuebles para ser demandados como viviendas u oficinas.

Como se comentó al principio, la participación de la iniciativa privada es fundamental en el proceso de renovación urbana, por lo mismo, uno de los objetivos más importantes de la intervención pública consiste en reducir las barreras que limitan su incorporación y en crear condiciones que hagan económicamente más atractiva la inversión en la zona, sin afectar el valor patrimonial de la misma. Para incidir en el segundo aspecto, entre las acciones más utilizadas en el ámbito internacional se encuentran diversos tipos de incentivos fiscales, y en la siguiente sección se explica teóricamente el papel que se espera desempeñen y cómo han funcionado en la realidad.

\section{Incentivos fiscales en el proceso de renovación urbana}

Por medio de la política fiscal el sector público puede incidir tanto en los ingresos como en los gastos de la iniciativa privada y, a su vez, intentar 
generar incentivos para invertir en bienes inmuebles. Si bien es cierto que incrementar los ingresos privados no es un objetivo que se pueda alcanzar de forma directa, es posible lograrlo de manera indirecta mediante la inversión en proyectos de rehabilitación de la zona y de la coinversión en proyectos detonadores que atraigan a consumidores potenciales. Reducir los gastos de las personas físicas y morales, en cambio, es un propósito que el gobierno sí puede lograr de forma directa, con la implementación de incentivos fiscales que reduzcan la carga impositiva y generen un ahorro fiscal, tanto para oferentes como demandantes de estos inmuebles; su intención es modificar el comportamiento de las personas, induciéndolas a invertir o comprar en determinados sectores o regiones.

Aunque en el ámbito internacional la estrategia basada en incentivos fiscales se ha utilizado ampliamente para fomentar el desarrollo de una zona específica, hasta ahora aún existe controversia sobre su eficacia, tanto en el plano teórico como en el empírico. En la literatura sobre desarrollo económico es común que se considere que los incentivos se justifican sólo si cumplen con lo siguiente (Eisinger, 1988): a) cuando afectan las decisiones de ubicación de los agentes económicos y esto genera más inversiones y nuevos empleos, lo cual se espera produzca un incremento en la demanda local de bienes y servicios, e impulse mediante efectos multiplicadores, mayor crecimiento económico, b) cuando lo que se deja de cobrar vía impuestos es menor que los beneficios económicos que se generan como resultado de los incentivos, y $c$ ) la medida es eficaz si quienes atraen lo incentivos son diferentes de quienes ya tenían considerado invertir en la zona, ya que de lo contrario el costo fiscal sería innecesario. En relación con este último punto, es común que se recomiende que los incentivos se administren de manera discrecional, asignándose sólo a aquellos agentes que tengan las características deseables para la localidad. El énfasis en lo anterior tiene que ver con la evidencia de que los ahorros fiscales normalmente no son el principal factor que se considera en las decisiones de ubicación; tienen más peso otros aspectos relacionados con el costo de las transacciones (Peters y Fischer, 2004). Pero si los incentivos no son necesarios para atraer a los agentes a invertir en la zona objetivo, entonces la medida se concibe como un despilfarro de recursos. En la práctica es muy complicado determinar en realidad quién necesita las exenciones fiscales, ya que se tienen incentivos precisamente para demostrar que existe la necesidad incluso cuando no sea cierto, es por ello que, aun cuando se considera contraproducente, la medida se ofrece normalmente de manera generalizada a todo aquél que lo solicite (Middleton, 2001).

En relación con la comprobación empírica, diversos estudios hechos en diferentes países muestran evidencia de que los incentivos tienen efec- 
tos pequeños o nulos (Bartik, 1991; Williams, 2006, Middleton, 2001; entre otros). Para el caso de las empresas, la causa principal es que los impuestos representaban un porcentaje pequeño del costo total de operación, por tanto, incluso una reducción significativa de los impuestos se ve neutralizada por pequeńos cambios en factores asociados a sus costos. Es importante resaltar que en la medida en que los incentivos han pretendido incidir en las decisiones de ubicación donde las condiciones de las alternativas son más homogéneas, la estrategia ha sido más efectiva (Anderson y Wassmer, 2000), es decir, es más probable que las exenciones fiscales sean más efectivas cuando las decisiones sólo contemplen diversas ubicaciones dentro de una ciudad.

Es probable que este último aspecto sea lo que ha motivado que en los proyectos de revitalización de centros históricos, con frecuencia se recurra al uso de incentivos fiscales. En proyectos de renovación de centros históricos es común que para los inversionistas exista una barrera de entrada que se relaciona con expectativas de menores rendimientos esperados en el centro, en comparación con lo que se puede obtener en otras ubicaciones, el papel del estímulo fiscal es entonces cerrar la brecha entre los rendimientos de las distintas ubicaciones para impulsar la inversión en el centro; si por otro lado la barrera se asocia con riesgos financieros, entonces su papel es compensar al inversionista potencial.

En Europa, los alcances de las estrategias fiscales han tenido diferencias importantes entre los países miembros de la comunidad, aunque todos han debido solicitar autorización a una comisión especial para ponerla en práctica. Por ejemplo, en España los incentivos fiscales otorgados en proyectos de renovación urbana se basan principalmente en deducciones al impuesto sobre la renta por donaciones realizadas para rehabilitar o conservar el patrimonio histórico, así como en deducciones al mismo impuesto por concepto de rehabilitación de edificios, mantenimiento y reparación de tejados y fachadas y la mejora de infraestructuras de los bienes declarados patrimonio mundial por la UnESCO. Por otro lado, en Francia (European Report, 2006) se exenta a todas las pequeñas y microempresas del pago de contribuciones a la seguridad social, impuesto sobre la renta e impuesto a los bienes raíces en 29 zonas urbanas que se pretende revitalizar. Esquemas de apoyos fiscales a microempresas en zonas de ciudades con importante deterioro urbano, también se propusieron a finales de la década de los noventa en Inglaterra (DTLGR, 2002).

Estos incentivos se han reforzado por una clara y manifiesta intención de parte de los gobiernos locales europeos de invertir de forma importante en los proyectos de restauración y rehabilitación urbana, de esta manera, los incentivos fiscales en realidad únicamente han servido para re- 
forzar las expectativas que el sector privado ya había construido en relación con el proyecto.

En Estados Unidos, también desde hace algunas décadas, se han implementado diversos programas federales, como la Recovery Economic Tax Act de 1981, que proveen incentivos para renovar edificaciones antiguas modificando la velocidad de depreciación de las inversiones realizadas, así como otros esquemas que intentan motivar la adquisición de vivienda, mediante la aplicación de diversos tipos de créditos fiscales que permiten deducir del pago del impuesto sobre la renta, los intereses de hipotecas y los impuestos a la propiedad, cuyos efectos se han vislumbrado sólo muchos años después de implementadas las acciones (Voith, 1999). Además, gobiernos locales han modificado sus impuestos a la propiedad también con el propósito explícito de procurar incentivos para invertir en proyectos de renovación urbana. Tal es el caso de Pittsburgh, que en 1980 modificó su impuesto a la propiedad incrementando el peso del valor del terreno hasta cinco veces sobre el valor de las edificaciones para obtener la base gravable, lo cual incidió positivamente en la cantidad de construcciones que se realizaron durante toda la década de los ochenta (Oates y Schwab, 1997).

En el caso de México ha habido diferentes proyectos de rehabilitación de zonas centrales a lo largo del país, como en Puebla, Jalisco, Monterrey o Campeche, sin embargo, tanto por la envergadura como por la cantidad de programas y recursos asignados (Delgadillo, 2008), sin duda los más significativos son aquéllos que se implementaron en la ciudad de México para rescatar su centro histórico. ${ }^{5}$ Para llevar a cabo su transformación, en 1990 se creó el Fideicomiso del Centro Histórico, que se hizo cargo de coordinar este proyecto conservador de los valores de la zona, en el cual participaron el gobierno del Distrito Federal, el Instituto Nacional de Antropología e Historia, el Instituto Nacional de Bellas Artes, historiadores del arte, restauradores, arquitectos y urbanistas. A su vez, para estimular la participación privada se diseñó un paquete de incentivos fiscales, tanto federales como estatales; a partir de 1991, el gobierno de la ciudad expidió cada año un acuerdo en el que se establecían estímulos fiscales en apoyo de las obras de rehabilitación emprendidas por propietarios, inversionistas y algunas instituciones públicas, como la Universidad Nacional Autónoma de México y el Banco de México.

Los incentivos fiscales utilizados por parte del gobierno federal incluían: 1) la deducción inmediata de $100 \%$ de las inversiones en inmuebles localizados en el centro histórico, para efectos del cálculo del impuesto

${ }^{5}$ En 1987, la unEsco reconoció el centro histórico de la ciudad de México como patrimonio histórico de la humanidad. 
sobre la renta; 2) la reducción del valor imponible de un inmueble hasta por un máximo de $60 \%$ para efectos del pago del impuesto por su enajenación, con la condición de que el comprador se comprometiera a su rehabilitación, de lo contrario, el impuesto no pagado por el vendedor lo tendría que asumir el comprador; y 3) la reducción de 90\% del impuesto al activo durante cinco años después de la restauración o rehabilitación del inmueble.

Por parte del gobierno local, los incentivos fiscales incluyeron un subsidio de hasta $100 \%$ del impuesto predial y de los impuestos sobre adquisición de inmuebles por los derechos relacionados con la construcción, por inscripción en el registro público de la propiedad o del comercio y por conexión y uso de redes de agua y drenaje (Fox, 2005).

Como resultado de la estrategia, se estimó que en el lapso de 1991 a 2003 los incentivos fiscales ya habían sumado más de 100 millones de pesos, ${ }^{6}$ al tiempo que la inversión privada ascendía a poco más de 2,225 millones de pesos, beneficiando obras de rehabilitación en cerca de 600 inmuebles (Cruz, 2004). Cabe señalar que se realizó una entrevista con el director general de la Fundación del Centro Histórico de la Ciudad de México, A.C., la inmobiliaria privada más importante dentro del proyecto de rescate, y ante la pregunta específica sobre el principal incentivo para invertir en la zona, contestó que fue el precio al que se vendieron algunos inmuebles, por debajo incluso de su costo de construcción. Es decir, las condiciones del mercado ya eran atractivas para la participación privada, aunque aparentemente no suficientes. Al igual que en los países desarrollados, en el caso de la ciudad de México el avance logrado es resultado de la aplicación de un plan integral, que además de incluir un programa de estímulos fiscales, basa sus esfuerzos en la construcción de un enlace estratégico entre inversionistas, la comunidad y el gobierno, necesario para generar las expectativas que se requieren para que cada quien se vea incentivado a participar.

Es importante mencionar que hay casos en otras ciudades latinoamericanas donde este tipo de incentivos tuvo resultados limitados, pues en muchos países de la región las tasas impositivas son bajas, lo cual reduce el valor de los incentivos vinculados a exenciones fiscales. En muchos otros casos no hay una recaudación fiscal eficiente o las penas por evasión fiscal no son significativas.

${ }^{6}$ Monto calculado a precios constantes de 2002. 


\section{Atractivo financiero de los incentivos fiscales aplicados en Veracruz}

Como se comentó en la primera sección, en septiembre de 2005 se publicó un decreto para otorgar estímulos fiscales para rescatar las zonas de monumentos históricos de las ciudades de Mérida, Yucatán; Morelia, Michoacán y la ciudad y puerto de Veracruz.

Estos estímulos se enfocaron a generar incentivos para que a la población le resultara atractivo restaurar, comprar o vender bienes inmuebles de esa zona, siempre y cuando esto último se hiciera con el fin de que el nuevo dueño adquiriera el inmueble para restaurarlo.

De acuerdo con el decreto, los estímulos fiscales se proponen incentivar:

- La compra de bienes inmuebles considerando que "forma parte de la inversión el valor de la adquisición de la construcción, excluyendo el valor del terreno", al otorgar la posibilidad a los contribuyentes de deducir en forma inmediata y hasta por el 100\% las inversiones que efectúen en estos inmuebles.

- Las reparaciones y adaptaciones a dichos bienes, que impliquen adiciones o mejoras al activo fijo. En este caso, el contribuyente también podrá deducir de forma inmediata y hasta por el 100\% el gasto que haga en reparaciones.

- La venta de bienes inmuebles, cuando éstos sean objeto de restauración o rehabilitación por la persona que los adquiere dentro de los dos años siguientes a la fecha de su adquisición. En este caso, el vendedor podrá considerar que "el costo comprobado de adquisición actualizado del bien inmueble, después de efectuar las deducciones señaladas en la Ley del Impuesto sobre la Renta, será cuando menos el equivalente al 40\% del monto de la enajenación de que se trate".

- El decreto también contempla otorgar un estímulo fiscal en el impuesto al activo por las obras de restauración o rehabilitación en bienes inmuebles ubicados en los perímetros que establece el decreto, sin embargo, el impuesto al activo quedó derogado en la última reforma fiscal que aplicó a partir de enero de 2008 y que introdujo el IETU.

A pesar de todo lo anterior, a la fecha las inversiones privadas no han sido las esperadas, ${ }^{7}$ lo que generó dudas sobre el alcance de la estrategia,

\footnotetext{
${ }^{7}$ De acuerdo con información proporcionada por la Dirección del Centro Histórico de Veracruz, hasta mediados de 2008 no se había dado la respuesta esperada de la iniciativa privada, y de hecho no se había realizado ninguna solicitud para aplicar los incentivos fiscales.
} 
especialmente después de la incorporación del IETU, pues en principio es claro que reduce las posibilidades de deducción de impuestos para los inversionistas potenciales. Para corroborar los efectos de las exenciones fiscales y las modificaciones al marco fiscal, se consideró pertinente evaluar qué tan atractivos resultan dichos incentivos simulando los flujos de efectivo de dos hoteles, uno ubicado en el centro histórico y otro en una zona de mayor desarrollo.

La evaluación se llevó a cabo para empresas de servicios turísticos, ya que el centro histórico es uno de los espacios más importantes de la zona conurbada en materia de recreación y esparcimiento, y precisamente este sector ha sido el más dinámico de todos los sectores productivos en la zona, en los últimos años su producción ha crecido anualmente a tasas superiores a $80 \%,{ }^{8}$ lo que hace suponer que reduciría las diferencias entre las rentabilidades esperadas por los inversionistas, en comparación con las que pudieran tener para negocios de otros giros (desarrollo de comercios o de viviendas) que también se encuentren en las dos ubicaciones comparadas: el centro histórico y la periferia.

La evaluación se realizó mediante un análisis comparativo de los resultados financieros de una inversión de 10 millones de pesos que se aplicaron a la compra y adaptación de un bien inmueble, tanto en el centro histórico como en la zona de desarrollo de la periferia de la ciudad, ${ }^{9}$ para convertir ambos inmuebles en pequeños hoteles de 24 habitaciones cada uno. Para realizar el análisis se establecieron los siguientes criterios y supuestos:

- Con un monto idéntico de inversión para cada zona, se presentan tres escenarios con diferentes niveles de ventas cada uno, el escenario pesimista con ventas equivalentes a $40 \%$ de la capacidad instalada, el escenario conservador con ventas equivalentes a $60 \%$ y el optimista con $80 \%$. Cada uno de estos niveles de ventas permanece constante durante el año y se considera que el primer año,

\footnotetext{
${ }^{8}$ Plan Estratégico para la Revitalización del Centro Histórico de Veracruz, agosto de 2008.

${ }^{9}$ Partir de inversiones idénticas llevadas a cabo en diferentes zonas de la ciudad, permite aislar las diferencias que pueden surgir por distintos giros de negocio, por campańas publicitarias, o por cualquier otra estrategia que pudiera modificar las preferencias de los consumidores, con el fin de identificar claramente cómo afectan los incentivos fiscales la rentabilidad de la inversión en función de su ubicación. Cabe suponer que aunque las inversiones son idénticas y se aplican para obtener un pequeño hotel con el mismo número de habitaciones, podría haber diferencias en cuanto a las características de los inmuebles, la superficie que ocupa cada uno, así como la distribución de los servicios del hotel y las habitaciones al interior del mismo. Se asume que en el centro histórico el inmueble tiene mayores probabilidades de ser más antiguo y no contar con estacionamiento propio, sin embargo, estas diferencias no invalidan los supuestos establecidos ni el análisis realizado, sino que se ven reflejadas en las diferentes tarifas que ofrecen.
} 
por introducción, las ventas tanto en el centro histórico como en la periferia son $85 \%$ menores que los años siguientes.

- En función de la experiencia de los empresarios del lugar, se supone que las ventas en el centro histórico son en promedio $12 \%$ más bajas que las que se registran en la periferia de la ciudad, y que los precios fijados en esta última zona son entre 20 y $25 \%$ más altos que en el centro. La diferencia de precios obedece principalmente a la mayor demanda por servicios, en este caso de hotelería, en una zona de la ciudad que está más cerca de las plazas comerciales, centro de convenciones, mejores restaurantes y lugares de diversión, que cuenta con mejor acceso vehicular y sin problemas de estacionamiento, así como inmuebles más nuevos y modernos.

- Se aplica la misma estructura de gastos para cada una de las inversiones.

- Los flujos son crecientes a partir de una tasa de inflación de 3\% anual a julio de 2008 y para descontarlos, se emplea una tasa de $11 \%$ anual, equivalente al rendimiento esperado en un portafolio que estuviera formado en $85 \%$ por títulos de deuda y en $15 \%$ por títulos volátiles. ${ }^{10}$

La simulación financiera permitió identificar que a pesar de que los ingresos de la empresa ubicada en la periferia de la ciudad, por las razones mencionadas, pueden llegar a ser hasta $31 \%$ mayores que los ingresos de la empresa ubicada en el centro histórico, la deducción inmediata y al $100 \%$ de la inversión en el centro le da ciertas ventajas, como no pagar impuestos en el primer ańo del ejercicio y reducirlos a partir del segundo año en un porcentaje que varía en función del escenario que se trate, así como aplicar el crédito por deducciones para reducir la base del IETU, el cual también cobra una importancia diferente en cada escenario.

Las empresas que no están en el perímetro señalado por el decreto de incentivos fiscales y que, por tanto, no pueden apegarse a él, tienen sin embargo la posibilidad de deducir hasta $74 \%$ de la inversión realizada en construcciones, con base en un decreto expedido en el año 2002, en el que el gobierno federal otorga un estímulo fiscal en materia de deducción inmediata de bienes nuevos de activo fijo. Este decreto se estableció en el título vir "De los Estímulos Fiscales" de la Ley del Impuesto sobre la Renta, con la finalidad de fomentar la inversión y la creación de empleos en el país.

\footnotetext{
${ }^{10}$ La composición del portafolio que sirve como referencia y la tasa asociada al mismo que se ocupa para descontar la inversión a julio de 2008, la proporcionó el director de la sucursal de Veracruz de Grupo Bursátil Mexicano, s.A. de c.v., www.gbm.com.mx.
} 
El resultado de aplicar ambos decretos se presenta a continuación por escenarios:

a) Escenario optimista. Con niveles de ingresos equivalentes a $80 \%$ de la capacidad instalada y la aplicación del decreto de incentivos fiscales, la empresa del centro histórico puede evitar el pago de impuestos en el primer ejercicio y reducirlos en promedio hasta en $62 \%$, con respecto a la proporción impuestos/ingresos de la empresa ubicada en la periferia durante los tres años que tarda en amortizarse la pérdida generada en el primer año de operación. Esto se traduce para la empresa del centro histórico en una rentabilidad en promedio $17 \%$ mayor a la registrada durante los mismos ańos en la otra empresa, sin embargo, en cuanto se termina de amortizar la inversión, la rentabilidad se vuelve $13.4 \%$ mayor en la periferia, lo que da como resultado flujos de efectivo $49.27 \%$ mayores. En este escenario, la tasa interna de retorno (TIR) para los primeros cinco años de vida de ambos proyectos es de $6.6 \%$ para la empresa del centro histórico y de $14.8 \%$ para la otra. Comparando estas tasas con el costo de oportunidad del capital, que de acuerdo con los criterios arriba mencionados es de $11 \%$, se rechazaría el proyecto ubicado en el centro histórico. Lo anterior resulta evidente al calcular el tiempo de recuperación de cada una de las inversiones, que es de siete años en el centro histórico y de cinco en otra zona de mayor desarrollo en la ciudad.

b) Escenario conservador. La aplicación del decreto de incentivos fiscales con los niveles de ingreso que presenta este escenario, permite a la empresa del centro histórico no pagar ISR durante los primeros cuatro años de operación, sin embargo, con excepción del primer ejercicio sí paga IETu durante esos años debido a que el crédito por deducciones no alcanza a cubrir los niveles de flujo de efectivo, que sirven de base para calcular este impuesto. Durante estos ejercicios, el IETU representa $57 \%$ del total de impuestos. Una vez que se terminó de amortizar la pérdida del primer año, el total a pagar por concepto de ISR se vuelve mayor que el IETU, por tanto, este último se deja de pagar. La empresa ubicada en la periferia no paga ISR los dos primeros ańos de operación, sin embargo, con mayores niveles de ingresos y un menor porcentaje de inversión por amortizar, paga a partir del tercer año tres veces más que la relación impuestos/ingresos que paga la empresa del centro histórico. Esto da como resultado que a pesar de la diferencia de ingresos, la diferencia que hay en el tiempo de recuperación de ambas inversiones se reduce, pues en este escenario la empresa del centro histórico se recupera sólo un año después que el tiempo que tarda 
en recuperarse la inversión ubicada en la periferia. A un plazo de cinco años, ambos proyectos tendrían tasas internas de retorno negativas, hasta el noveno año estas tasas se vuelven aceptables en función del costo de capital.

c) Escenario pesimista. En este escenario, debido a que por menores niveles de ventas la pérdida del primer año es mayor, la amortización de la misma en los siguientes ejercicios se prolonga por un mayor número de años, lo que elimina para ambas inversiones el pago del ISR en los primeros ejercicios, durante tres años para la inversión en la periferia y siete ańos para la inversión en el centro histórico. Sin embargo, la inversión en la periferia, a diferencia de la del centro histórico, paga IETU, pues el crédito por deducciones no alcanza a cubrir sus flujos de efectivo, lo que no sucede en el centro, donde por seis años no se pagan ninguno de los dos impuestos. El resultado es que durante siete años, del segundo al octavo, la opción de la periferia paga hasta seis veces más impuestos que la empresa del centro histórico. Una vez amortizada la pérdida, a partir del noveno año de operación, esta ventaja impositiva se elimina y ambas inversiones pagan ISR y no IETU porque este último resulta menor que el primero. A pesar de la ventaja fiscal, la inversión en el centro histórico tardaría más de 20 años en recuperarse y en la periferia sólo tardaría catorce.

\subsection{El efecto del impuesto empresarial a tasa única (IETU)}

Cuando en 2005 se emitió el decreto de incentivos fiscales para estimular la inversión en el centro histórico, no se había establecido el pago del IETU, que entró en vigor en enero de 2008 y obliga a las personas físicas y morales a pagarlo por los ingresos que obtengan por la enajenación de bienes, prestación de servicios independientes y por el otorgamiento del uso o goce temporal de bienes. Este impuesto se calcula aplicando la tasa de $16.5 \%$ para el ejercicio de 2008 , de $17 \%$ para 2009 y de $17.5 \%$ para el ejercicio de 2010 y subsecuentes, a la cantidad que resulte de disminuir el total de los ingresos percibidos por las actividades antes descritas, menos las deducciones autorizadas por la ley en el artículo 5, capítulo II "De las Deducciones” de la Ley del Impuesto Empresarial a Tasa Única. Con el resultado, el contribuyente tiene la obligación de hacer su balance entre este impuesto y el impuesto sobre la renta, y pagará al fisco el que genere un gravamen mayor.

El resultado de la implementación de este impuesto a las condiciones que se habían establecido a partir del decreto de estímulos fiscales es que, 
a mayores niveles de ingreso generados por la inversión en el centro histórico, se reduce el ahorro fiscal que pudiera lograrse en el pago del ISR.

Con ingresos equivalentes a $80 \%$ de la capacidad instalada, el pago del IETU representa en promedio $68 \%$ del total de los impuestos pagados y $37.6 \%$ de lo que hubiera sido el total a pagar por concepto de ISR, si no hubieran existido los estímulos fiscales. En la medida en que los ingresos se reducen, el impacto del IETU disminuye también, es decir, en el escenario conservador, la representación del IETU en el pago total de impuestos disminuye a $57 \%$, y es importante hacer notar que a los niveles de ingreso que maneja este escenario, no se paga ISR durante los primeros cuatro años de vida del negocio, pero sí se paga el IETU, que representa $23.38 \%$ de lo que se hubiera pagado por IsR, es decir, la ventaja fiscal que dan los incentivos sobre el ISR se reduce por la implementación del IETU en $62.4 \%$ en el escenario optimista y en $76.6 \%$ en el conservador.

Con ingresos equivalentes a $50 \%$ de la capacidad instalada no se paga IETU ni ISR en los primeros siete años de operación del negocio ubicado en el centro histórico, porque los flujos de efectivo son menores a las amortizaciones pendientes. Cuando finaliza la aplicación de este crédito fiscal, casi al mismo tiempo que la pérdida del ejercicio del primer año se ha terminado de amortizar también, el ISR que se comienza a pagar es mayor al IETU, por tanto, bajo los supuestos que plantea este escenario, este último no se paga en ningún año. La empresa ubicada en la periferia en cambio paga IETU durante los tres primeros ejercicios e ISR a partir del cuarto ańo de operación, pues sus flujos de efectivo no llegan a ser menores a las amortizaciones pendientes.

En resumen, se puede apreciar la eficacia de los incentivos fiscales en la reducción de la carga impositiva de las empresas, cuando éstas tienen ingresos iguales o menores a los que se obtienen con ventas equivalentes a $50 \%$ de su capacidad instalada, pues a bajos niveles de ingreso es posible gozar de las ventajas que ofrecen los incentivos al reducir la base gravable del ISR y que este beneficio no se reduzca por el pago del IETU. En la medida en que los ingresos se incrementen, el pago del IETU aumenta su proporción en el total de impuestos pagados por la empresa y reduce la ventaja fiscal que trata de proporcionar el decreto de incentivos.

\section{Comentarios finales}

El trabajo conjunto de los sectores privado y público se propone desde hace unos años como vía para la preservación sostenible del medio urbano, al permitir que las limitaciones que ambos presentan por separado, en cierta medida se vean mitigadas. Como señala Rojas (2006), mientras que ambos comparten riesgos, uno aporta su visión, su compromiso a 
largo plazo y su capacidad de coordinación, y el otro su experiencia en el mercado y la capacidad de poder gestionar varios proyectos respondiendo a la demanda existente (Rojas, 2006).

No obstante, aunque es evidente que los resultados de las estrategias de renovación urbana implementadas en Veracruz sólo se apreciarán en el largo plazo, con el análisis previo se hace evidente que no existe esa colaboración eficiente entre el sector público y el privado, y es posible identificar los aspectos más relevantes que afectan el desarrollo de una sintonía ideal entre las motivaciones e intereses de los principales actores de la iniciativa privada y el sector público, los cuales podrían ser las causas de la débil respuesta de los primeros ante las acciones emprendidas por los segundos. Por tanto, para alinear los incentivos es importante considerar lo siguiente:

1) Pareciera que para la iniciativa privada no existen expectativas reales de que se pueda revertir la tendencia de deterioro y abandono que sufre el centro, lo cual incrementa el riesgo de su inversión en esa zona. Una de las razones que generan esta expectativa es la lógica de que el éxito del proyecto requiere que el gobierno municipal invierta en el proceso de recuperación del centro histórico, y que esta recuperación es un proyecto de largo plazo, plazo que no es alternativa para las administraciones públicas que tienen periodos de tres ańos, durante los cuales requieren alcanzar resultados que les permitan conservar el voto de la ciudadanía.

2) La posibilidad de atraer consumidores con mayor poder económico se reduce por el problema del tráfico vehicular y el estacionamiento, que es creciente en esta zona y que provoca que la gente evite visitar el centro. Esto se presenta como barrera fundamental que determina la posibilidad de crecimiento de la rentabilidad de la zona.

3) Finalmente, los estímulos fiscales no garantizan una rentabilidad equivalente a la que se podría obtener en otras zonas de mayor desarrollo de la ciudad, ya que en realidad los incentivos no preveen siquiera qué tan grandes son las brechas de los rendimientos entre las diferentes ubicaciones. Los incentivos tampoco se han enfocado a modificar el interés por demandar espacios en cada uno de sus usos potenciales, y en particular para vivienda, pues de origen están diseñados para revitalizar el mercado inmobiliario sin que exista ninguna estrategia encaminada a retener y recuperar la importante función habitacional que tuvo hace décadas el centro de la ciudad. Además, la reforma fiscal de 2008 que implementó el IETU para todas las personas físicas o morales, reduce el potencial de las exenciones debido a que los estímulos fiscales que se decre- 
taron antes de la aprobación de esta ley, ofrecen deducciones que reducen la base gravable del ISR pero no del IETU. De las experiencias analizadas se puede verificar que los incentivos fiscales juegan un papel importante para proteger los bienes patrimoniales, por lo que resulta conveniente, como mínimo, mejorar el régimen tributario de las ayudas percibidas para su conservación y restauración, así como disminuir el gravamen derivado de la mera titularidad de dichos bienes. A pesar de la importancia que tiene la política fiscal en apoyo a la inversión privada en los proyectos de rehabilitación del centro histórico, la experiencia evidencia las limitaciones que puede tener esta estrategia si no se inscribe en un proyecto rector sustentado en un plan que contemple opciones de financiamiento acordes a cada una de sus etapas.

\section{Bibliografía}

Adair, Alaistar, Jim Berry, Stanley McGreal y Arlene Quinn (2002), "Factor affecting the level and form of private investment in regeneration", informe de Office of the Deputy Prime Minister, Londres.

Adair, Alaistar, Jim Berry y Stanley McGreal (2006), "Institutional investment in regeneration: Necessary conditions for effective funding", research report, www.bura.org.uk/OneStopCMS/Core/ CrawlerResourceServer.aspx? resource $=2$ F6C8AE6-FD74-4542B036-F0E5B52FD723\&mode=link\&guid=74e4b9167f7b4705 819870 b8c40c01ea, 6 de octubre de 2008.

Anderson, John y Robert Wassmer (2000), Bidding for business: the effcacy of local economic development incentives in a metropolitan area, W. E. Upjohn Institute for Employment Research, Michigan.

Bartik, Timothy (1991), Who benefits from state and local economic development policy, W. E. Upjohn Institute for Employment Research, Michigan.

Berry, Jim, Alaistar Adair, Stanley McGreal y Joanna Poon (2003), "Benchmarking urban regeneration", Research Report, rpp.ulster.ac. uk/research/reports/benchmarking.pdf, 10 de septiembre de 2008.

Carrasco, Gustavo (2007), "Santiago de Chile: propuesta para la revitalización y recuperación de un centro urbano", documento de 
trabajo Sirchal 2.2, www.uchilefau.cl/institutos/invi/extension/ pdfs/07-11-29_carrasco_santiago.pdf, 20 de septiembre de 2008.

Carrión, Fernando (2000), "Lugares o flujos centrales: los centros históricos urbanos", Publicaciones de cepal, Santiago de Chile, www. cepal.org/publicaciones/xml/7/6557/LCL1465PE. pdf\#search $=\% 22$ Lugares $\% 20$ o\%20flujos\%20centrales $\% 3 \mathrm{~A} \% 20$ los\%20centros\%20hist\%C3\%B3ricos\%20urbanos\%22, 28 de septiembre de 2009.

Cruz Martínez, Ángel Bernardo (2004), "Proyecto de sustentabilidad integral del centro histórico de la ciudad de México", Grupo Ciudad y Patrimonio, A.C., imaginarios.com.mx/redmcs/syp/iv/ centros_historicos/mesa5/angel_bernardo_cruz_martinez.pdf, 28 de septiembre de 2009.

Delgadillo Polanco, Víctor (2008), "Repoblamiento y recuperación del centro histórico de la ciudad de México, una acción pública híbrida, 2001-2006", Economía, Sociedad y Territorio, viII (28), El Colegio Mexiquense, Toluca, pp. 817-845.

DTLGR (Department for Transport, Local Government and the Regions) (2002), "Modernising local government finance: A green paper", informe de Office of the Deputy Prime Minister, Londres.

Eisinger, Peter (1988), The rise of the entrepreneurial State, University of Wisconsin Press, Madison.

Espinosa, Ana Isabel (2004), "Amenazas y nuevas estrategias del comercio de centro urbano. El caso de Alicante", Boletín de la AGE, 38, Alicante, pp. 153-174.

European Report (2006), "Inner cities: Commission clears french urban regeneration scheme", European social policy (Goliath), goliath. ecnext.com/coms2/gi_0199-7477317/INNER-CITIES-COMMISSION-CLEARS-FRENCH.html\#abstract, 24 de octubre de 2008.

Fox, Catherine (2005), Alianzas tripartitas. Reconocimiento del tercer sector, Banco Interamericano de Desarrollo, Washington. 
Ihlandfeldt, Keith (1995), "The importance of the central city to the regional and national economy: A review of arguments and empirical evidence", Cityscape: A Journal of Policy Development and Urban Development, 1 (2), US Department of Housing and Urban Development, Washington, pp. 125-150.

Leal, Eusebio (2004), "La rehabilitación del Centro Histórico de La Habana: una obra esencialmente humana", Forum Universal de las Culturas-Barcelona 2004. Diálogo sobre el Turismo, la Diversidad Cultural y el Desarrollo Sostenible", Barcelona, 14-16 de julio, www.biospherehotels.org/tourism2004/pdf/final/EusebioLeal.pdf, 20 de mayo de 2006.

McGreal, Stanley, Alaistar Adair, Jim Berry, Bill Deddis y Suzanne Hirst (2000), "Accessing private sector finance in urban regeneration: Investor and non-investor perspectivas", Journal of Property Research, 17 (2), Routledge, Londres, pp. 109-131.

McGreal, Stanley Jim Berry, Greg Lloyd y John McCarthy (2002), “Taxbased mechanism in urban regeneration: Dublin and Chicago Models", Urban Studies, 39 (10), Routledge, Londres, pp. 18191831.

Middleton, Luke (2001), "Literature Review: Tax abatements and economic development incentives", Policy Research Institute, The University of Kansas, Report No. 49, Kansas.

Oates, Wallace y Robert M. Schwab (1997), "The impact of urban land taxation: The Pittsburgh experience", National Tax Journal, 50 (1), National Tax Association, Washington, pp. 1-21.

Papke, James (1995), "Interjurisdictional business tax-cost differentials: convergence, divergence and significance”, State Tax Notes, 9 (24), Tax Analysts, Washington, pp. 1701-1711.

Peters, Alan y Peter Fisher (2002), State enterprise zone programs: Have they worked?, W. E. Upjohn Institute for Employment Research Press, Michigan.

Peters, Alan y Peter Fisher (2004), "The failure of economic development incentives", Journal of the American Planning Association, 70 (1), American Planning Association, Chicago, pp. 27- 37. 
Programa de ordenamiento y revitalización del Centro Histórico de la Ciudad de Veracruz (1998), Gobierno del Estado de Veracruz, Xalapa.

Programa de ordenamiento, mejoramiento y revitalización del Centro Histórico de la Ciudad de Veracruz (2006), Gobierno del Estado de Veracruz, Xalapa.

Rojas, Eduardo (2004), Volver al centro: la recuperación de áreas urbanas centrales, Banco Interamericano de Desarrollo, Washington.

Rojas, Eduardo (2006), "La preservación del patrimonio histórico urbano en América Latina y el Caribe. Una tarea de todos los actores sociales", Banco Interamericano de Desarrollo, Washington, www. iadb.org/sds/doc/SOC125sp.pdf, 12 de agosto de 2008.

Rodríguez, Arantxa y Elena Martínez (2001), "Del declive a la revitalización: oportunidades y límites de las nuevas políticas urbanas en Bilbao", Ciudad y Territorio: Estudios Territoriales, xxxiII (129), Ministerio de Vivienda, Madrid, pp. 441-459.

SHCP (Secretaría de Hacienda y Crédito Público) (2003), "Decreto por el que se otorga un estímulo fiscal en Materia de Deducción Inmediata de Bienes Nuevos de Activo Fijo", Diario Oficial de la Federación, México.

SHCP (Secretaría de Hacienda y Crédito Público) (2005), "Decreto por el que se otorgan estímulos fiscales para el rescate de zonas de monumentos históricos de la ciudad de Veracruz", Diario Oficial de la Federación, México.

Vázquez, Daniel (2007), "Aspectos a considerar en la propuesta de peatonalización en el centro histórico del puerto de Veracruz", Revista de la Universidad Cristóbal Colón, cuarta época, año 1, 22, Veracruz, pp. 60-69.

Voith, Richard (1999), "Does the federal tax treatment of housing affect the pattern of metropolitan development?", Business Review, Federal Reserve Bank of Philadelphia, Filadelfia, pp. 3-16. 
Williams, Brendan (2006), "Fiscal incentives and urban regeneration in Dublin", Working Paper pep 06/01, Planning and Environmental Policy, University College Dublin, pp. 1-17.

Recibido: 25 de mayo de 2009. Reenviado: 14 de diciembre de 2009. Aceptado: 4 de enero de 2010.

Daniel Vázquez-Cotera. Es doctor en finanzas públicas por la Universidad Veracruzana, maestro en economía por la Universidad Autónoma de Nuevo León y licenciado en economía por la Universidad Cristóbal Colón. Actualmente es profesor-investigador adscrito al departamento de Investigación de la Universidad Cristóbal Colón en Veracruz. Su línea actual de investigación es: procesos de revitalización en centros históricos. Entre sus publicaciones recientes destacan: "Aspectos a considerar en la propuesta de peatonalización en el centro histórico del puerto de Veracruz", Revista de la Universidad Cristóbal Colón, cuarta época, 22, Veracruz, pp. 60-69 (2007); "Capacidad y uso de los estacionamientos en el centro histórico de Veracruz”, Revista de la Universidad Cristóbal Colón, cuarta época, 22, Veracruz, pp. 97-109 (2007). Durante 2008 fue consultor economista para el Banco Interamericano de Desarrollo y el H. Ayuntamiento de Veracruz en el estudio sobre el potencial económico y financiero del centro histórico de Veracruz.

Elena Moreno-García. Es doctora en ciencias económicas y administrativas por la Universidad de Almería, España; maestra en finanzas por la Universidad de las Américas, Puebla, y licenciada en economía por la Universidad Cristóbal Colón. Actualmente es directora de la licenciatura en economía de la Universidad Cristóbal Colón y candidata del Sistema Nacional de Investigadores (sNI). Su línea actual de investigación es: irreversibilidad de la inversión inmobiliaria. Entre sus publicaciones recientes destacan: "Análisis y Clasificación de las explicaciones a la crisis financiera mexicana de 1994", Eseconomía. Revista de la Escuela Superior de Economía del Instituto Politécnico Nacional, 15, IPN, México, pp. 29-52 (2007); "Análisis de las políticas y programas económicos implementados en México en la década de los ochenta: antecedentes de la crisis financiera de 1994", Revista de la Universidad Cristóbal Colón, tercera época, 21, año III, pp. 83-94 (2005). Durante 2008 fue asesor financiero en el estudio sobre el potencial económico y financiero del centro histórico de Veracruz. 